\title{
MDM2 promotes epithelial-mesenchymal transition and metastasis of ovarian cancer SKOV3 cells
}

\author{
Ying Chen ${ }^{1,3}$, Dan-Dan Wang ${ }^{1,3}$, Ye-Ping Wu ${ }^{1}$, Dan Su ${ }^{2}$, Tian-Yi Zhou ${ }^{1}$, Ren-Hua Gai ${ }^{1}$, Ying-Ying Fu ${ }^{1}$, \\ Lin Zheng ${ }^{1}$, Qiao-Jun $\mathrm{He}^{1}$, Hong Zhu ${ }^{*, 1}$ and Bo Yang ${ }^{\star, 1}$ \\ 'Zhejiang Province Key Laboratory of Anti-Cancer Drug Research, Institute of Pharmacology \& Toxicology, College of \\ Pharmaceutical Sciences, Zhejiang University, Hangzhou 310058, China and '2Zhejiang Cancer Hospital, Hangzhou 310022, China
}

Background: Metastasis accounts for the most lethal reason for the death of ovarian cancer patients, but remains largely untreated. Epithelial-mesenchymal transition (EMT) is critical for the conversion of early-stage ovarian tumours into metastatic malignancies. Thus the exploration of the signalling pathways promoting EMT would open potential opportunities for the treatment of metastatic ovarian cancer. Herein, the putative role of MDM2 in regulating EMT and metastasis of ovarian cancer SKOV3 cells was investigated.

Methods: The regulatory effects by MDM2 on cell motility was emulated by wound-healing and transwell assays. The effects on EMT transition and Smad pathway were studied by depicting the expression levels of epithelial marker E-cadherin as well as key components of Smad pathway. To evaluate the clinical relevance of our findings, the correlation of MDM2 expression levels with the stages of 104 ovarian cancer patients was investigated by immunohistochemistry assay.

Results: We demonstrate that MDM2 functions as a key factor to drive EMT and motility of ovarian SKOV3 cells, by facilitating the activation of TGF- $\beta$-Smad pathway, which results in the increased transcription of snail/slug and the subsequent loss of E-cadherin levels. Such induction of EMT is sustained in either E3 ligase-depleted MDM2 or E3 ligase inhibitor HLI-373-treated cells, while being impaired by the N-terminal deletion of MDM2, which is also reflected by the inhibitory effects against EMT by Nutlin-3a, the $\mathrm{N}$-terminal targeting agent. The expression levels of MDM2 is highly correlated with the stages of the ovarian cancer patients, and the higher expression of MDM2 together with TGFB are closely correlated with poor prognosis and predict a high risk of ovarian cancer patients.

Conclusions: This study suggests that MDM2 activates Smad pathway to promote EMT in ovarian cancer metastasis, and targeting the N-terminal of MDM2 can reprogram EMT and impede the mobility of cancer cells.

Ovarian cancer is the second-most common gynaecological cancer, and ranks as the most lethal gynaecological malignancy worldwide (Jayson et al, 2014; Siegel et al, 2015). The major reason for the high death rate is the widely metastatic spread predominantly in the abdomen (Bowtell et al, 2015; Narod, 2016). Epithelialmesenchymal transition (EMT) is a major process for the conversion of early-stage ovarian tumours to invasive and metastatic malignancies, promoting the aggressiveness of ovarian cancers, due to the loss of epithelial characteristics and acquisition of mesenchymal characteristics (Nieto et al, 2016). Therefore, EMT was described as critical event in cancer progression and metastasis (Tsai and Yang, 2013), and has drawn much attention in ovarian

\footnotetext{
*Correspondence: Dr H Zhu; E-mail: hongzhu@zju.edu.cn or Dr Professor B Yang; E-mail: yang924@zju.edu.cn

${ }^{3}$ These authors contribute equally to this work.
}

Received 24 May 2017; revised 5 July 2017; accepted 17 July 2017; published online 17 August 2017

(C) 2017 Cancer Research UK. All rights reserved 0007-0920/17 
cancer metastasis research (Rosano et al, 2014). EMT is generally induced by developmental signalling pathways, most notably, the TGF- $\beta$ pathway is regarded as a primary inducer of EMT (Xu et al, 2009; Tsai and Yang, 2013). In response to TGF- $\beta$, the type II receptor $(\mathrm{T} \beta \mathrm{RII})$ kinases phosphorylate and activate the type I receptors $(\mathrm{T} \beta \mathrm{RI})$, which in turn phosphorylates Smad2 and Smad3. Phosphorylated Smad2 and Smad3 then form trimers with Smad4, and the complex translocates to the nucleus to regulate target gene transcription, in large part via transcription factors Snail family, leading to the molecular changes, including the loss of epithelial markers, such as E-cadherin, and the upregulation of mesenchymal markers, like Fibronectin, N-cadherin etc (Xu et al, 2009). Moreover, emerging evidences have indicated that TGF- $\beta$ aberrantly expresses in ovarian cancer and triggers metastasis and malignancy (Matsumura et al, 2011). Hence, blockage of TGF$\beta$-Smads-Snails pathway to resist EMT may be an effective strategy to cure the metastatic ovarian cancer.

The human homologue of murine double minute-2 (MDM2) is an oncogene related with various types of malignances, including the occurrence of metastasis in several reports (Wang et al, 2009; Zheng et al, 2013). MDM2 mainly functions as an E3 ligase targets various substrates (generally tumour suppressors, such p53 and $\mathrm{Rb}$ ) for ubiquitylation and proteasome-dependent degradation, thereby promoting cancer malignancy. Recently, several lines of evidences implicate the crosstalk of MDM2 and Smad pathway. It was reported that TGF- $\beta 1$-activated Smad3/4 transcription factors, leading to increased MDM2 protein expression and destabilisation of p53. In addition, activated Smad3 and MDM2 were both positive in $65 \%$ of the metastatic late-stage breast cancer, demonstrating a strong correlation between TGF- $\beta$ induced MDM2 and metastatic tumours (Araki et al, 2010). Moreover, in our previous study, we found that a potent and selective small molecular MDM2 antagonist Nutlin3 inhibited TGF- $\beta 1$-induced EMT in ovarian cancer cells (Wu et al, 2014b), indicating the correlation of MDM2 and EMT in ovarian cancer. A recent study demonstrated that MDM2 induced EMT by enhancing Snail expression (Lu et al, 2016); however, whether MDM2 is involved in the TGF- $\beta$-Smad signalling pathway thus promoting the progression of EMT remains unclear.

In this study, we find that MDM2 facilitated cell motility and EMT occurrence in ovarian cells through the activation of TGF- $\beta$ Smads and subsequently increased Snail/Slug transcription. The depletion of MDM2 impaired TGF- $\beta$ induced EMT through TGF$\beta$-Smads-Snail/Slug pathway. Further studies showed that the $\mathrm{N}$-terminus (1-109) of MDM2 is required for inducing EMT, since only Nutlin-3, the antagonist targeting the N-terminus of MDM2, could impede the cell migration. In the contrast, HLI-373, an ubiquitin E3 ligase inhibitor targeting the C-terminus of MDM2, failed to impose effects on the Snail/Slug expression and rescue TGF- $\beta$-induced cell motility. These findings suggest that MDM2 may be a potent therapeutic target of ovarian cancer and MDM2 $\mathrm{N}$-terminal selective antagonist could be regarded as an effective strategy for the metastatic ovarian cancer therapy.

\section{MATERIALS AND METHODS}

Cell culture and reagents. The human ovarian cancer cell line SKOV3 and the mouse Lewis lung cancer (LLC) cells were obtained from Shanghai institute of Biochemistry and Cell Biology, Chinese Academy of Medical Sciences (Shanghai, China). SKOV3 cells were maintained in RPMI-1640 medium (Gibco, Grand Island, NY, USA), LLC was cultured in DMEM, supplemented with $10 \%$ foetal bovine serum (HyClone, Logan, UT, USA) and standard antibiotics at $37^{\circ} \mathrm{C}$ in a humidified atmosphere containing $5 \% \mathrm{CO}_{2}$.
TGF- $\beta 1$ was purchased from R\&D systems (Minneapolis, MN, USA). To induce EMT, cells were planted on the previous day, starved (serum-free medium) overnight and treated with $5 \mathrm{ng} \mathrm{ml}^{-1}$ TGF- $\beta 1$. Nutlin-3 was purchased from Sigma-Aldrich (St Louis, MO, USA). HIL-373 was obtained from Tocris Bioscience (Bristol, UK).

Gene transfection and RNA interference technology. The pCMV-myc3-MDM2 plasmid or small interfering RNA was transfected into cells using Oligofectamine (Invitrogen, 12252011) or Lipofectamine 2000 (Invitrogen, 11668-019) according to the manufacturer's instructions. The pCMV-myc3-MDM2 plasmid was purchased from Addgene (plasmid \#20935, Cambridge, MA, USA). And the deletion mutants were constructed by Genscript Co. Ltd (Shanghai, China). Human MDM2 siRNA was produced by GenePharma Co. Ltd (Shanghai, China). The sequences of human siRNAs were as follows, si-MDM2-1: sense: $5^{\prime}$-AAUGCC UCAAUUCACAUAGAUTT- $3^{\prime}$, antisense: $5^{\prime}$-AUCUAUGUGA AUUGAGGCAUUTT-3'; si-MDM2-2: sense: $5^{\prime}$-GCCUGGCUCU GUGUGUAAUTT- $3^{\prime}$ and antisense: $5^{\prime}$-AUUACACACAGAGC CAGGCTT-3'.

Western blotting analysis. Western blotting analysis of proteins in cell lysates was determined as previously described (Wu et al, 2014b). Primary antibodies used were as follows: anti-MDM2 (Cat\# 556353), anti-E-cadherin (Cat\# 610405) and anti-Fibronectin (Cat\# 610078) were purchased from BD (San Diego, CA, USA); anti-Slug (Cat\# 9585), anti-Smad2/3 (Cat\# 3102), anti-p-Smad2 (Cat\# 3108), anti-p-Smad3 (Cat\# 9520), anti-Smad4 (Cat\# 9515) and anti-myc-tag (Cat\# 2278) were from Cell Signaling Technology (Beverly, MA, USA); anti-Snail (Cat\# AF3639) and anti-TGF-beta RI (Cat\# AF3025) were from R\&D; anti- $\beta$-Actin (Cat\# sc-47778) was purchased from Santa Cruz (Santa Cruz, CA, USA).

Transwell assay and wound-healing assay. Transwell and wound-healing assay analysis of cell motility were determined as previously described (Wu et al, 2014b). A Boyden Chamber system (Costar Corp., Cambridge, MA, USA) was used for migration assay. SKOV3 cells (starved-treated overnight) were seeded in a density of $3 \times 10^{4}$ per well in the upper chamber with serum-free medium, while serum was placed in the lower chamber. After 24hour incubation, the cells remaining in the upper chamber were removed with cotton buds, and then the cells had migrated through the chamber were fixed with $75 \% \mathrm{EtOH}$ for $30 \mathrm{~min}$ followed by $0.1 \%$ crystal violet staining for $20 \mathrm{~min}$. The stained cells were subsequently photographed by microscope (LEICA DMI $4000 \mathrm{~B})$. For the analysis, an equal volume of $10 \%$ acetic acid was added to each well to completely dissolve the stained crystal violet. OD750 was read to quantify the percentage of migrated cells.

For wound-healing assay, the SKOV3 cells overexpressed MDM2 or knockdown of MDM2 were seeded in 24-well plates in complete culture medium and grown to confluence. Cells were serum starved overnight and then wounded by scratching with a sterilised $200 \mu$ l pipette tip and then cultured with fresh complete culture medium for indicated times. After that, cells were photographed using a LEICA DMI 4000B microscope.

Immunofluorescence. Cells transfected with pCMV $\beta$-myc3MDM2 plasmid or empty vector were plated in 6-well plates. Then cells were fixed with $4 \%$ paraformaldehyde in PBS for $15 \mathrm{~min}$, and blocked with 3\% bovine serum albumin (Sigma-Aldrich, A7906) and $0.3 \%$ Triton X-100 in PBS for $1 \mathrm{~h}$ at room temperature. MDM2 and p-Smad3 was probed with primary antibodies anti-MDM2 (1:50) and anti-pSmad3 $(1: 150)$ in $\mathrm{PBS}$ overnight at $4{ }^{\circ} \mathrm{C}$ at room temperature and Alexa Fluor 488 donkey anti- rabbit IgG secondary antibody (Invitrogen, A-11008) or Alexa Fluor 568 donkey antimouse IgG secondary antibody (Invitrogen, A-10037) diluted in $1: 500$ in PBS for $1 \mathrm{~h}$ at $37^{\circ} \mathrm{C}$. Nuclei were visualised by staining with DAPI (4' 6-diamidino-2-phenylindole; Sigma-Aldrich, D9542). 
Cells were than washed thrice with PBS and imaged with Leica DMI 400B fluorescence microscope.

Anti-MDM2 (Cat\# sc-965), anti-E-cadherin (Cat\# sc-7870) were purchased from Santa Cruz (Santa Cruz), and anti-p-Smad3 (Cat\# 9520) was from Cell Signaling Technology (Beverly, MA, USA).

Real-time PCR. Analyses of mRNA levels using real-time PCR was determined as previously described (Wu et al, 2014b). The sequence of oligonucleotide primers E-cadherin (forward primer: 5'-TTCTGCTGCTCTTGCTGTTT-3'; reverse primer: $5^{\prime}$-TGG CTCAAGTCAAAGTCCTG-3'), Slug (forward primer: $5^{\prime}$-GAAA GGCCTTCAACTGCAAA- $3^{\prime}$; reverse primer: $5^{\prime}$-TGACATCTGA GTGGGTCTGG-3'), Snail (forward primer: 5'-AGATGCATA TTCGGACCCAC-3'; reverse primer: $5^{\prime}$-CCTCATGTTTGTG CAGGAGA- $3^{\prime}$ ) and GAPDH (forward primer: $5^{\prime}$-GTCATCCATG ACAACTTTGG-3'; reverse primer: $5^{\prime}$-GAGCTTGACAAA GTGGTCGT-3') were synthesised by Sangon (Shanghai, China).

Luciferase reporter assay. The procedure for the SBE-luciferase reporter assay has been previously described (Wu et al, 2014b). SKOV3 cells were seeded in 96-well plates at 5000 cells per well and transfected with MDM2 expression or empty Vector plasmid. After 24-h transfection, Lipofectamine plus reagent (Invitrogen) was used to perform co-transfection of pGL3 SBE-luciferase reporter construct (Addgene) along with pRL-TK Renilla. After 24$\mathrm{h}$ incubation, cells were treated with or without TGF- $\beta 1$ $\left(5 \mathrm{ng} \mathrm{ml}^{-1}\right)$ for $6 \mathrm{~h}$, and then harvested and assayed for SBEluciferase activity, which was measured using using the Dualluciferase Reporter Assay System (Promega, Madison, WI, USA) as described in the manufacturer's instructions. Each luciferase assay was performed 3 times in triplicate and values represented as mean \pm s.d. determined by Student's $t$-tests.

Immunohistochemistry. Formalin-fixed and paraffin-embedded tissue sections from human ovarian cancer tissues or normal tissues were deparaffinisation, and treated with $3 \%$ hydrogen peroxide in methanol for $15 \mathrm{~min}$ at room temperature to quench endogenous peroxidase activity. Then the slides were microwaved (10 min) in $0.01 \mathrm{M}$ citrate buffer $(\mathrm{PH} 6.0)$ at $95-100{ }^{\circ} \mathrm{C}$ and cooled down at room temperature for $20 \mathrm{~min}$ for antigen retrieval and this progress was repeated at least 3 times. After antigen retrieval, the slides were blocked using 20\% normal goat serum and then stained with MDM2 antibody overnight followed by biotinylated secondary antibodies and horseradish peroxidase-conjugated avidin. The sections were then stained with $\mathrm{DAB}$ and the nuclei were counterstained using haematoxylin. Anti-MDM2 (Cat\# 556353) were purchased from BD (San Diego, CA, USA), haematoxylin was from Beyotime Biotechnology (Shanghai, China). MDM2 visualised with 3, 3'-diaminobenzidine.

Statistical analysis. Student's $t$-tests were used to validate the significance of the data analysed and considered statistically significant when $P<0.05\left({ }^{\star} P<0.05\right.$, ${ }^{* *} P<0.01$ and $\left.{ }^{* * *} P<0.001\right)$.

\section{RESULTS}

MDM2 is correlated with the motility of SKOV3 cells. To evaluate whether MDM2 is correlated with the motility in SKOV3 cells, we introduced exogenous myc-MDM2 plasmid and silenced the expression of MDM2 using siRNA respectively to validate the motility using transwell assay and wound-healing assay. The effects of transfection were shown in Figure 1A. The transwell assay was used to validate the chemotaxis of SKOV3 cells influenced by MDM2, and we found that the cells with MDM2 overexpression invaded migration onto the lower surface of the porous membrane (Figure 1B), while siMDM2 with 2 different sequences distinctly reversed foetal bovine serum-induced migration (Figure 1D).
Similar findings were confirmed by wound-healing assay. As shown in Figure 1C and E, overexpression of MDM2 induced cells to migrate and enhanced the closure of 'wounding area' of cells, in the contrast, siMDM2 significantly reduced cell migration remained cell-free space after $24 \mathrm{~h}$ compared with control group cells. These findings indicated the correlation between MDM2 and the motility of ovarian cancer cells.

Knockdown of MDM2 impairs the TGF- $\beta$-induced EMT of SKOV3 cells. Since EMT plays a crucial role in early steps of metastasis, we further detected whether MDM2 was associated with EMT of SKOV3 cells. After transfected with pCMV $\beta$-MDM2 for $48 \mathrm{~h}$, the morphology of SKOV3 cells switched to mesenchymal appearance, with the loss of intercellular connections (Figure 2A), accompanied with the loss of epithelial marker E-cadherin (Figure 2B).

Mounting evidences showed that TGF- $\beta$ is among the strongest inducer of physiological and pathological EMT, which is mediated by binding to its receptor to activate the downstreaming transcription factors. In this study, we treated SKOV3 cells with TGF- $\beta\left(5 \mathrm{ng} \mathrm{ml}^{-1}\right)$ to stimulate the occurrence of EMT. As shown in Figure 2C, TGF- $\beta$ induced the cells to acquire mesenchymal-like morphology, however, which was subsequently rescued by the knockdown of MDM2 using siRNA, with the establishment of cell adhesion and polarity. Consistent with these results, siMDM2 prevented the downregulation of E-cadherin protein levels induced by TGF- $\beta$ stimulation, indicating the acquisition of epithelia properties (Figure 2D and Supplementary Figure S1A). Afterwards, we introduced consistent activated TGF- $\beta$ receptor I (CA-TBRI) to mimic TGF- $\beta$ treatment, resulting in the raised expression of mesenchymal marker Fibronectin. In accordance with previous findings, the MDM2 knockdown depressed the Fibronectin expression caused by CA-TBRI (Supplementary Figure S2). However, the MDM2 depletion failed to reversed the reduction of E-cadherin protein leaded by nuclear factor- $\kappa \mathrm{B}$, another reported EMT inducer, induced by TNF- $\alpha$ treatment (Supplementary Figure S1B), indicating the enhanced EMT induced by MDM2 is TGF- $\beta$ dependent.

Since the transcription of E-cadherin can be repressed by TGF- $\beta$ induced EMT, we next conducted real-time PCR analysis to evaluate the mRNA level of E-cadherin. As shown in Figure 2E and $\mathrm{F}$, compared with vector controls, MDM2 overexpression impeded the E-cadherin mRNA level, while MDM2 deletion significantly abolished the reduction caused by TGF- $\beta$. Collectively, these results suggested the MDM2 knockdown depressed the TGF- $\beta$ induced EMT of ovarian cancer cells.

MDM2 depletion reverses the TGF- $\beta$-induced Snail/Slug activation. Aforementioned data indicated that MDM2 depletion could rescued the attenuated E-cadherin protein and mRNA levels in response to TGF- $\beta$ treatment. Accumulating evidences showed that TGF- $\beta$ stimulation of EMT represses the synthesis of E-cadherin transcripts in large part via its ability to induce the expression of the Snail family, including Snail1 and Snail2 (Slug). Therefore, we are interested whether MDM2 mediated the protein and mRNA levels of Snail family. As shown in Figure 3A and B, exogenous MDM2 was transfected into SKOV3 cells, and enhanced not only the protein expression but also the mRNA levels of Snail and Slug. Furthermore, when MDM2 was depleted in cancer cells, followed by co-incubation with TGF- $\beta$, the TGF- $\beta$ induced upregulation of Snail/Slug protein and mRNA levels was significantly attenuated by MDM2 knockdown (Figure 3C and D).

It is interesting to note that several certain responses exhibited in response to TGF- $\beta$ are governed primarily by the expression of various Smad2/3-interacting transcription factors, as well as their association with additional transcriptional activators or receptors. To further investigate whether the raised Snail/Slug mRNA is attributed to the interaction with receptor-activated Smads 
A

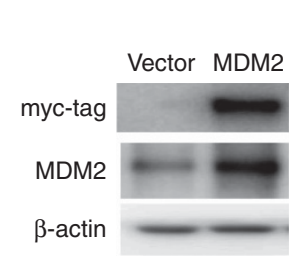

B

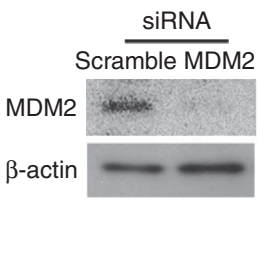

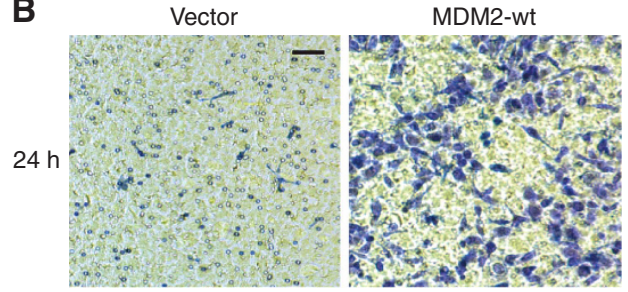

D

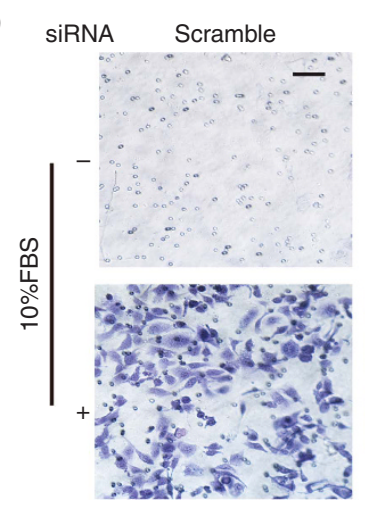

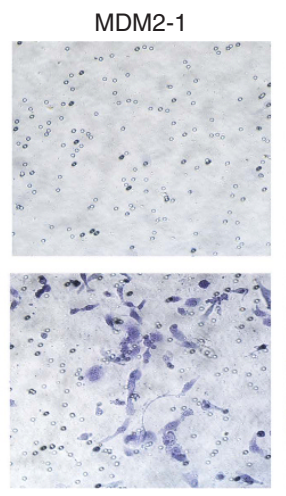

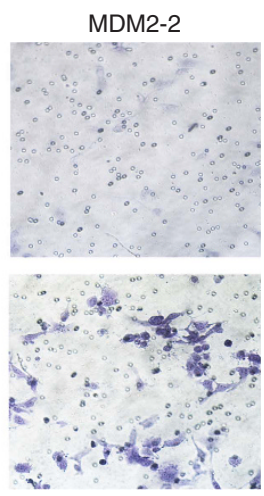

C
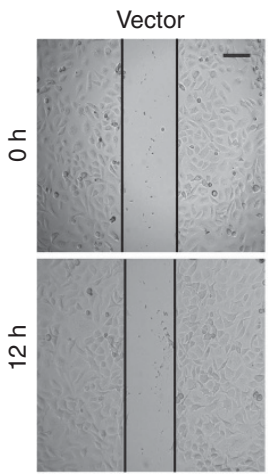
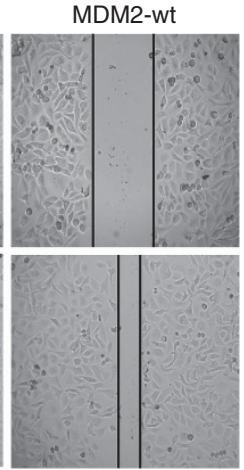

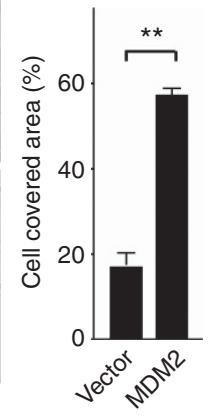

E
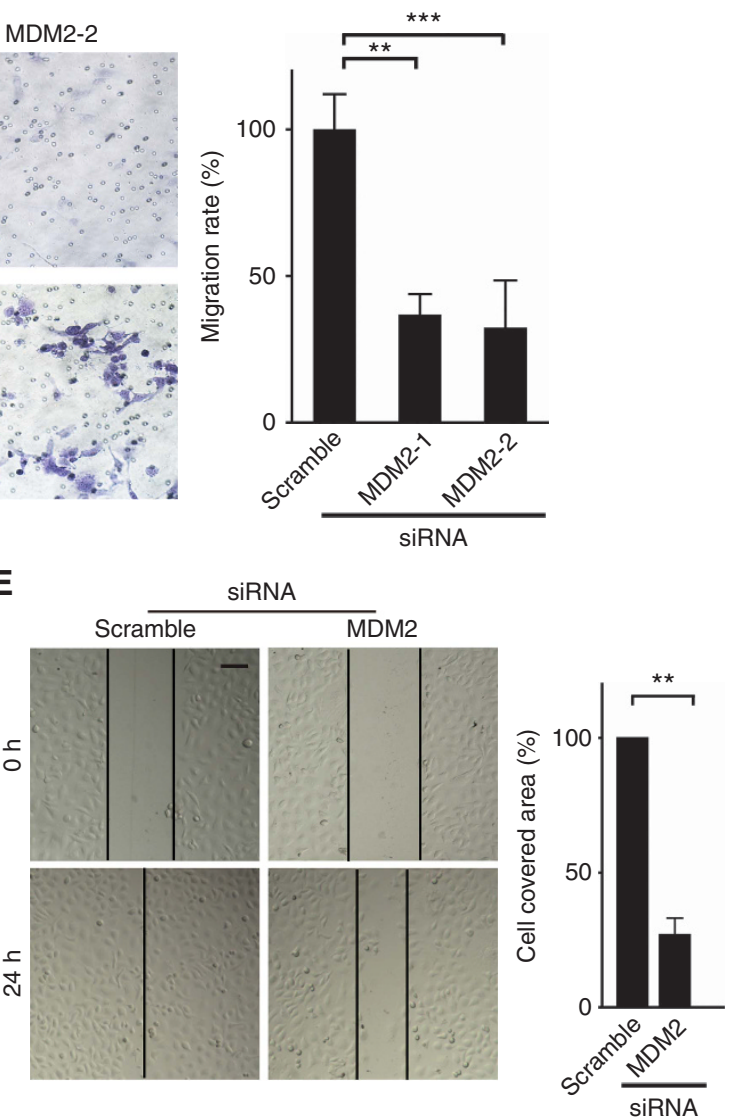

Figure 1. MDM2 enhanced the motility of ovarian cancer cells. (A) The MDM2 overexpression and knockdown were achieved by the transfection of SKOV3 cells with PCMV $\beta$-MDM2 plasmid and MDM2-targeting siRNA, respectively. (B) Overexpression of MDM2 enhanced the ability of migration and invasion by transwell assay after incubation for $24 \mathrm{~h}$, scale bar $=50 \mu \mathrm{m}$. (C) Wound-healing assay showed the result that the woundhealing ability of SKOV3 cells was increased by exogenous MDM2 transfection, scale bar $=50 \mu \mathrm{m}$. (D) The MDM2 silencing using siRNA resulted in reduced ability of invasion, which was determined by transwell assay. Solubilise crystal violet with $200 \mu 10 \%$ acetic acid per well, and quantify migratory rate by measuring the OD. Data were presented as means \pm s.d. from three independent experiments. ${ }^{\star \star} P<0.01 ;{ }^{\star \star \star} P<0.001$, as evaluated using Student's t-test. (E) Knockdown of MDM2 reduced cells motility, which was carried out by wound-healing assay.

(R-Smads), we introduced Smad promoter fragment SBE-luciferase as well as renilla plasmids in the presence or absence of MDM2 knockdown. As shown in Figure 3E, MDM2 deficiency resisted the SBE-luciferase activity upregulated by TGF- $\beta$, suggesting that MDM2 regulated Smads transcriptional activity.

These data collectively illustrated MDM2 enhanced the transcription and protein expression of Snail/Slug through the Smads transcriptional activity.

MDM2 facilitated Smad2/3 activation, which is conversely indispensable for MDM2 to induce EMT. To define the role of MDM2 for Smad activation, we firstly overexpressed MDM2 by introducing the MDM2 plasmid into the SKOV3 cells and detected the phosphorylation level of Smad2/3 by Western Blot. As shown in Figure 4A, MDM2 overexpression triggered the upregulation of pSmad2 (S465/467) and pSmad3 (S423/425), even without the treatment of TGF- $\beta$, indicating that MDM2 could activate Smad pathway by increasing the phosphorylating Smad2. Interestingly, the additional TGF- $\beta$ into MDM2 transfected cells failed to further increase $\mathrm{pSmad} 2$, thus raising the possibility that MDM2 converged with TGF- $\beta$ pathway to activate the Smad complex. The result of immunofluorescence assay further displayed that MDM2 induced the pSmad3 expression (Figure 4D). Furthermore, the MDM2 silencing resulted in restrain of $\operatorname{Smad} 2 / 3$ activation irritated by TGF- $\beta$ (Figure $4 \mathrm{~B}$ ). Interestingly, we further found that the Smads pathway was essential for MDM2 to induce EMT, conversely. Depletion of Smad2, Smad3, and Smad4 respectively rescued downregulation of E-cadherin protein induced by MDM2 overexpression (Figure 4C). Altogether, these results suggested MDM2 facilitated Smad2/3 activation, which is conversely indispensable for MDM2 to induce EMT.

$\mathrm{N}$-terminal of MDM2 is required for inducing EMT and promoting the motility of SKOV3 cells. Given the critical role of MDM2 in inducing EMT, we hypothesised that MDM2 inhibitors could inhibit EMT and the motility of ovarian cancer 
A

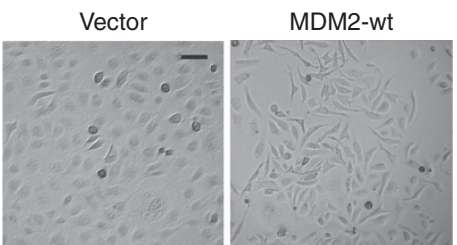

C

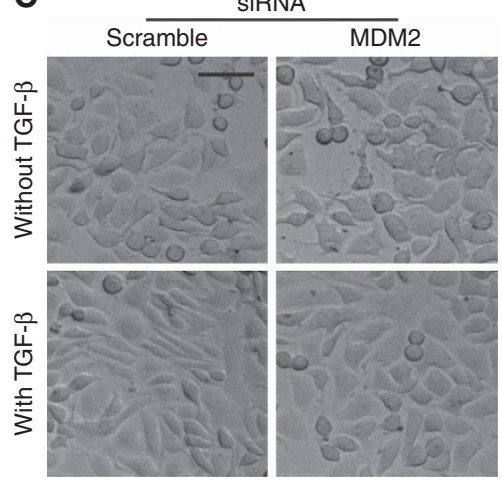

B

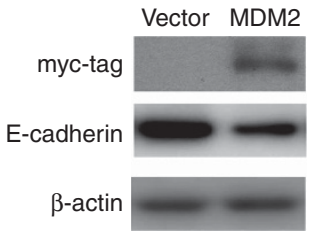

E

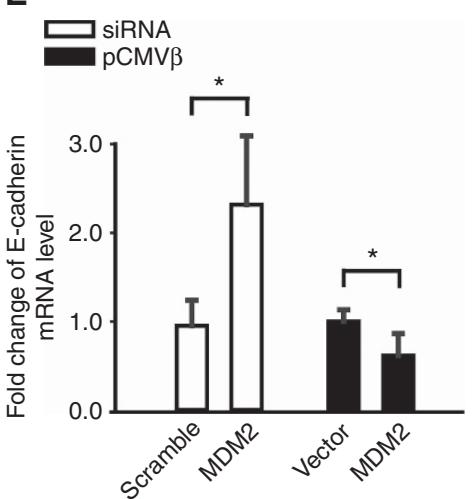

D

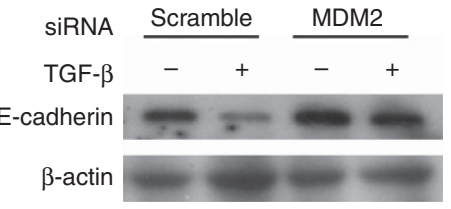

$\mathbf{F}$
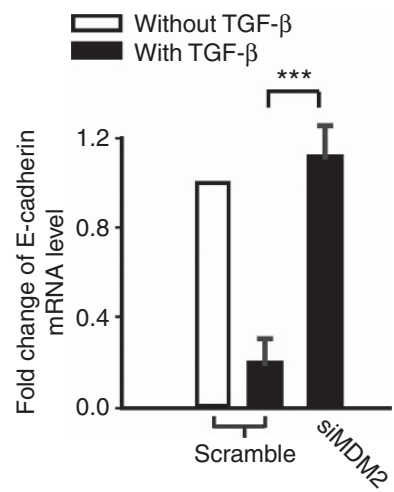

Figure 2. MDM2 induced EMT in ovarian cancer cells. (A) Overexpression of MDM2 caused morphologic changes to a mesenchymal phenotype in SKOV3 cells. Representative photos were as indicated, scale bar $=50 \mu \mathrm{m}$. (B) Transfection of MDM2 induced downregulation of E-cadherin protein levels, which is determined by western Blot. (C) The TGF- $\beta\left(5 \mathrm{ng} \mathrm{ml}^{-1}\right)$ treatment stimulated morphologic changes to mesenchymal phenotype, which is subsequently rescued by MDM2 depletion, scale bar $=50 \mu \mathrm{m}$. (D) Knockdown of MDM2 increased E-cadherin protein expression, and abrogated the loss of E-cadherin upon TGF- $\beta$ treatment. (E) The mRNA levels of E-cadherin expression were monitored by qRT-PCR assay. The MDM2 exogenous transfection resisted the E-cadherin transcription, whereas MDM2 siRNA upregulated the E-cadherin mRNA levels. (F) The MDM2 knockdown reversed the decline of E-cadherin mRNA triggered by TGF- $\beta$. Data were presented as means \pm s.d. from three independent experiments. ${ }^{\star} P<0.05 ;{ }^{\star * \star} P<0.001$, as evaluated using Student's t-test.

A

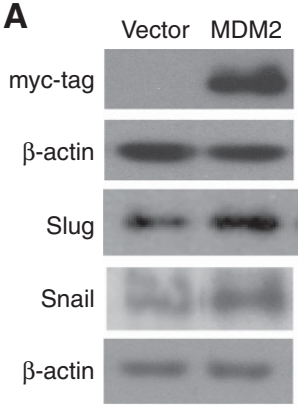

D

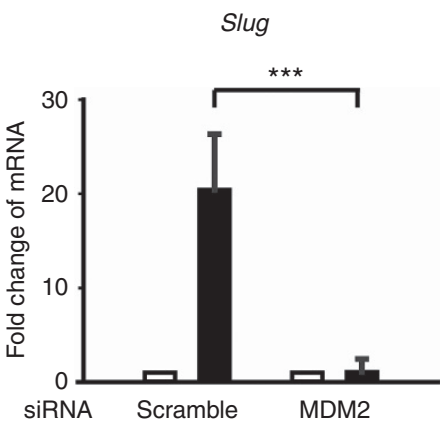

B
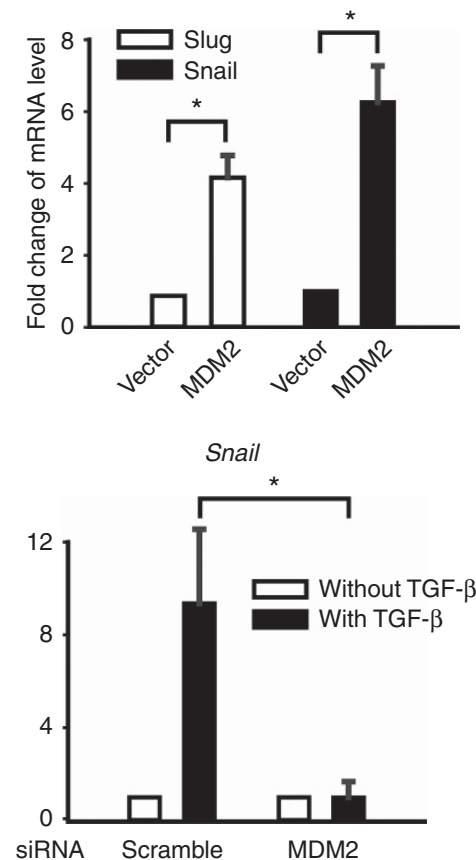

C

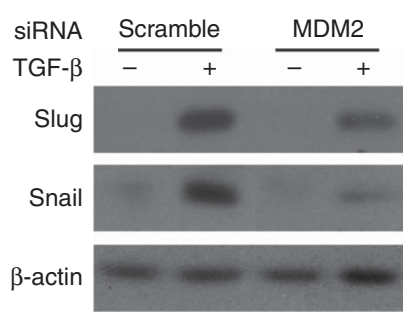

E

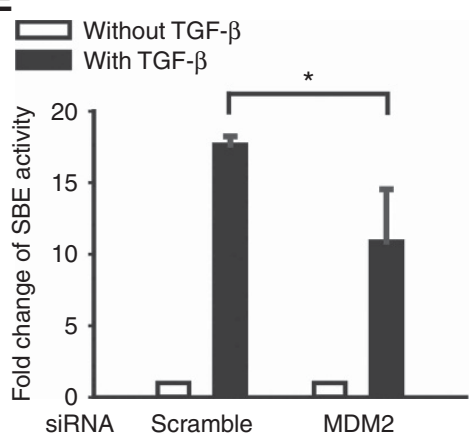

Figure 3. MDM2 regulated the TGF- $\beta$-induced Snail/Slug activation in ovarian cancer cells. (A) MDM2 plasmid was introduced to SKOV3 cells and raised the protein expression of Snail and Slug. (B) The qRT-PCR analyses revealed that the mRNA levels of Snail and Slug were upregulated by MDM2 overexpression. (C) The MDM2 siRNA abrogated the induction of Snail and Slug protein by TGF- $\beta\left(5 \mathrm{ng} \mathrm{ml}{ }^{-1}\right.$ ). (D) MDM2 knockdown significantly repressed the mRNA levels of Snail and Slug increased by TGF- $\beta$. (E) SKOV3 cells treated with scramble or MDM2 siRNA were transfected with plasmids encoding SBE-luciferase and pRL-IRES Renilla in the presence or absence of TGF- $\beta\left(5 \mathrm{ng} \mathrm{ml}^{-1}\right)$ for $24 \mathrm{~h}$. MDM2 depletion depressed the TGF- $\beta$-induced-SBE activity, which was measured by Luciferase assay. Data were presented as means \pm s.d. from three independent experiments. ${ }^{\star} P<0.05 ;{ }^{* \star \star} P<0.001$, as evaluated using Student's t-test. 
A

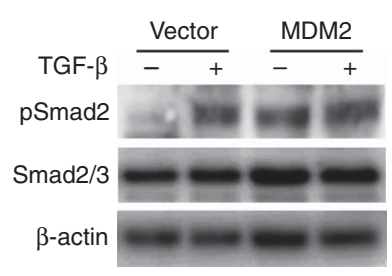

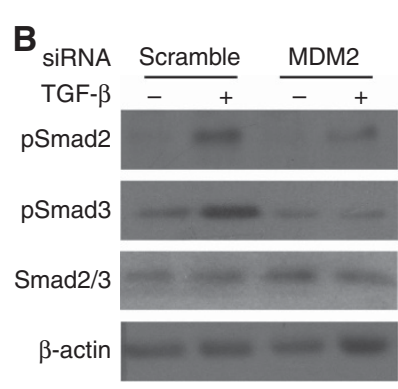

pSmad3

D
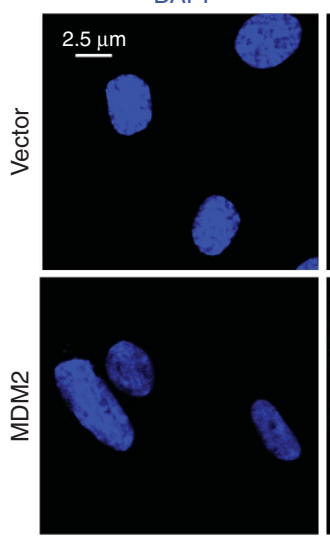
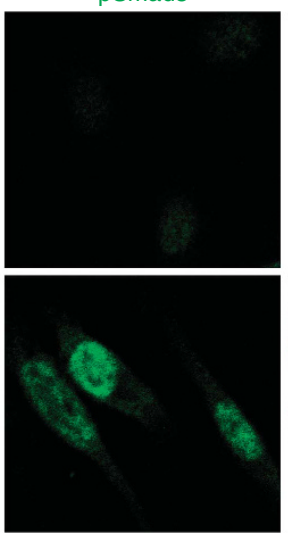

MDM2
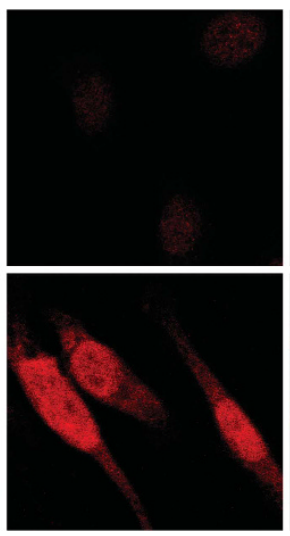
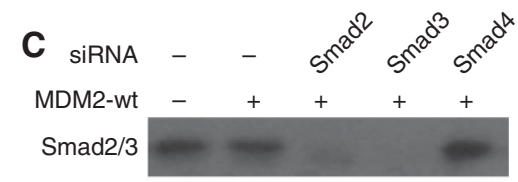

Smad4

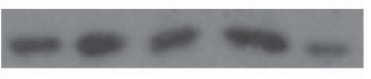

E-cadherin

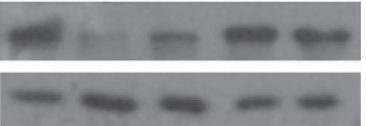

$\beta$-actin
pSmad3/MDM2
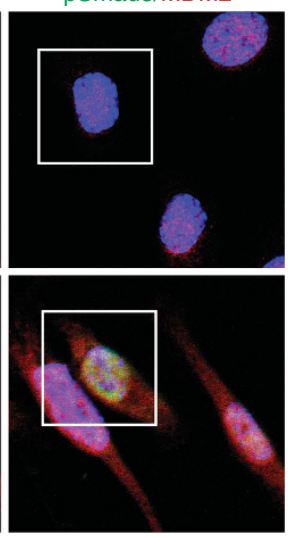

pSmad3/MDM2
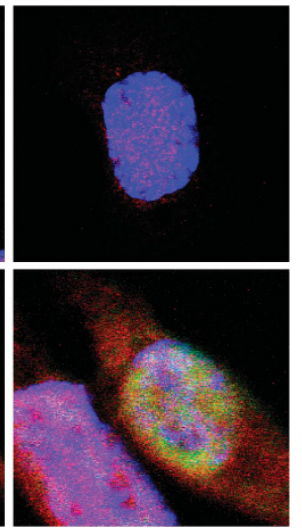

Figure 4. MDM2 facilitated Smad2/3 activation, which was conversely indispensable for MDM2 to induce EMT. (A) MDM2 overexpression induced the phosphorylation of Smad2/3, even without the treatment of TGF- $\beta$. (B) MDM2 siRNA attenuated pSmad2 and pSmad3 levels provoked by TGF- $\beta$ treatment $\left(5 \mathrm{ng} \mathrm{ml}^{-1}\right.$ ). (C) Depletion of Smad2, Smad3 or Smad4 rescued the downregulation of E-cadherin protein induced by MDM2 overexpression. (D) The expression of pSmad3 (green) and MDM2 (red) were detected by immunofluorescence, and MDM2 overexpression significantly enhanced pSmad3 expression in SKOV3 cells.

cells. In line with this, our previous study found Nutlin-3, a MDM2 antagonist, inhibited EMT by interfering with TGF- $\beta 1$-Smad Snail/ Slug axis ( $\mathrm{Wu}$ et al, 2014b). Similar observations were also observed in the present study: Nutlin-3a decreased the phosphorylation of Smad2/3, accompanied by the reduced expression of Snail/Slug protein levels (Figure 5A), and exhibited significant inhibitory effects against TGF- $\beta$-induced migration of SKOV 3 cells (Figure 5B). In addition, the in vivo anti-metastatic ability of Nultin-3a was also observed in LLC allograft models of C57BL/6 mice (Supplementary Figure S3).

Unexpectedly, not all the MDM2 inhibitors possessed the ability to suppress cancer cell migration: HLI-373, a MDM2 inhibitor interrupting its ubiquitin E3 ligase activity, could abolish the ubiquitylation of its substrate protein p53 (Supplementary Figure S4A), but failed to rescue the upregulation of Snail/Slug and the activation of Smad2 induced by TGF- $\beta$, along with the E-cadherin mRNA levels repressed by TGF- $\beta$ (Figure 5C and Supplementary Figure S4B). Moreover, HLI-373 failed to impose inhibitory effects on the motility of ovarian cancer cells (Figure 5D). Similar results were obtained when we used exogenous MDM2 overexpression to trigger the ability of invaded migration, which was not affected by the HLI-373 co-incubation (Figure 5E).

To gain insight into the mechanisms underlying the distinct effects of these two MDM2 inhibitors, we supposed that the MDM2 region targeted may play differential roles in the EMT, as Nutlin-3 targets the N-terminus of MDM2 binding to p53, while HLI-373 targets the C-terminus functioning as an E3 ubiquitin ligase. Therefore, we introduced four MDM2 plasmids fused to a myc-tag into SKOV3 cells. Consistent with our hypothesis, only the mutant of MDM2- $\Delta 1-109$ (Deletion of the 1-109-residue amino-terminal domain in MDM2 N-terminus) lost the ability to decrease the E-cadherin protein levels, while a C-terminal
RING-finger domain mutant with deletion of residues 429491(MDM2- $4422-491$ ), and a RING finger mutant with cysteine changed to alanine leading to loss of its E3 ligase activity (MDM2 C464A), exerted similar effects as that of the full-length wide-type MDM2 (Figure 5F). Transwell assay further showed that the induction of migration was impaired by the deletion of MDM2 $\mathrm{N}$-terminus (Figure 5G).

Collectively, these data suggested that the $\mathrm{N}$-terminal, but not the E3 ligase activity was required for MDM2 to induce EMT and increase the motility of ovarian cancer cells, and the small molecule inhibitors targeting $\mathrm{N}$-terminal of MDM2 could be regarded as potential strategies to interfere with EMT and the subsequent metastasis.

MDM2 expression is associated with the stage of ovary carcinoma. The overexpression of MDM2 in ovarian cancer has been reported in several previous studies. Similar results were obtained in our present study: immunohistochemistry assay revealed in 104 ovarian cancer cases, 94\% were detected to demonstrate positive expression of MDM2, while MDM2 was negative expressed in all normal tissues cases (Figure 6A and B). To further determine the connection between MDM2 expression and ovarian cancer, we used log IOD in Image-Pro Plus 6.0, illustrating that MDM2 expression levels from ovarian cancer were significantly higher than normal tissues (Figure 6C). Since ovarian cancer is a highly metastatic cancer, most ovarian cancer patients harbour metastasis upon initial diagnosis. We next detect the correlation of MDM2 expression and metastasis of ovarian cancer. As shown in Table 1, all 18 cases with high expression of MDM2 were from stages III and IV ovarian cancer patients with metastasis. Among the all 104 ovarian cancer cases with MDM2 expression, only 21 cases were from stages I and II non-metastatic patients. A positive 
A

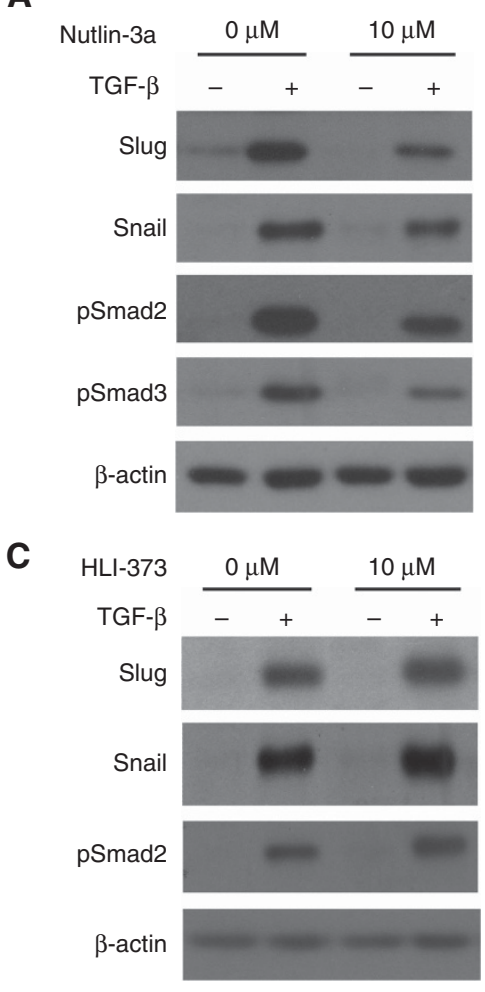

$\mathbf{F}$

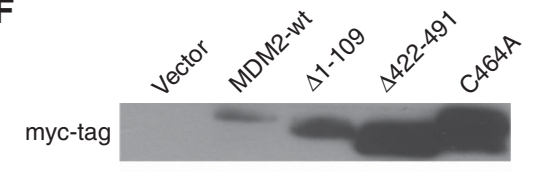

E-cadherin

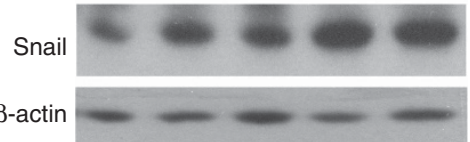

B

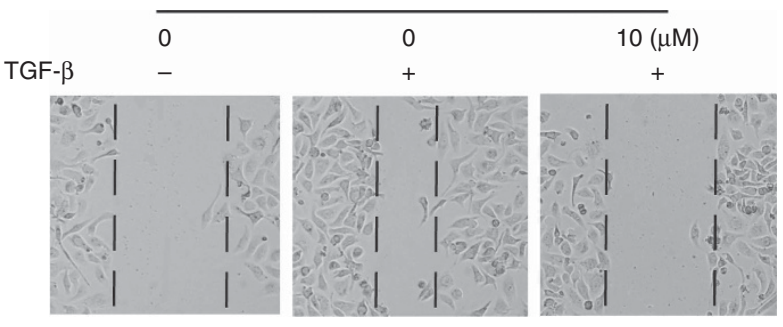

D

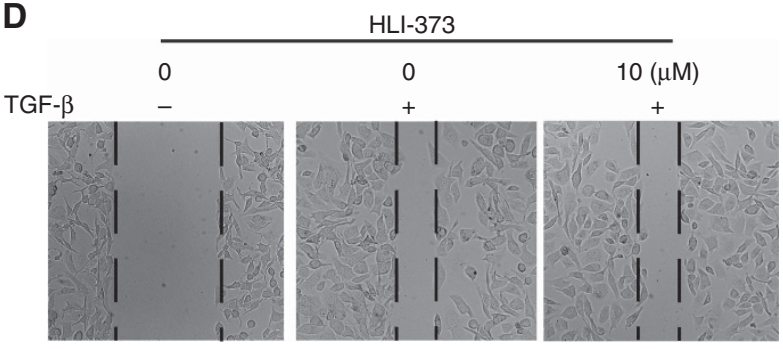

E

HLI-373

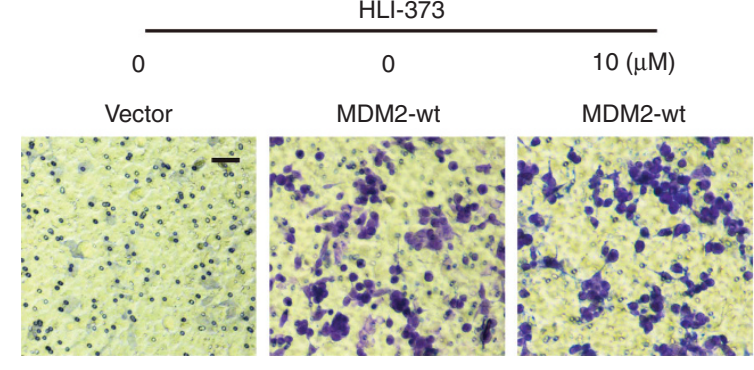

G

Vector

MDM2-wt

$\Delta 1-109$
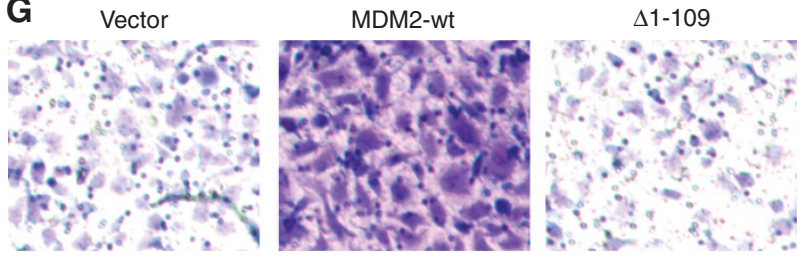

Figure 5. N-terminal of MDM2 is required for inducing EMT and the motility of ovarian cancer cells. (A) Nutlin-3a repressed the expression of Snail and Slug protein levels and the phosphorylation of Smad2/3 induced by TGF- $\beta\left(5 \mathrm{ng} \mathrm{ml}^{-1}\right)$. (B) Wound-healing assay showed Nutlin-3a greatly reversed TGF- $\beta$-mediated migration. (C) HLI-373 failed to rescue the protein expression of Snail, Slug and p-Smad2 raised by TGF- $\beta$ stimulation. (D) TGF- $\beta$-mediated wound-healing could not be repaired by HLI-373. (E) Transwell assay revealed that HLI-373 imposed no effect on the ability of invaded migration triggered by exogenous MDM2 plasmid transfection. (F) Mutated MDM2 ( $\Delta 1-109)$ lost the activity to promote the reduction of E-cadherin, while MDM2 ( 4 422-491) and MDM2 (C464A) mutants exhibited similar pattern with that of wide-type MDM2. (G) The deletion of MDM2 N-terminus failed to induce cell migration.

correlation between the MDM2 expression and ovarian cancer metastasis is also found with statistically significant $(P=6.13 \mathrm{E}-05)$. These results suggest that MDM2 expression levels are associated with the stage and metastasis of ovary carcinoma.

\section{DISCUSSION}

MDM2 has been reported to be amplified and overexpressed in a variety of human malignances (Zheng et al, 2013; Wu et al, 2014a; Inoue and Fry, 2016), and closely associated with cancer metastasis (Shi et al, 2009), contributing to the poor prognosis and short survival in patients(Grochola et al, 2011). In line with these reports, the present study found that MDM2 overexpression was observed in $94 \%$ of ovarian cancer patients, and the high levels of MDM2 were associated with the stage and metastasis of ovary carcinoma (Figure 6 and Table 1). In our study, we also found that the MDM2 depletion arrested cell proliferation of SKOV3 cells (Supplementary Figure S5), indicating that MDM2 plays a significant role in human ovarian cancer. However, the mechanisms underlying cancer metastasis mediated by MDM2 remains unclear (Shadfan et al, 2012). Yang et al (2006) suggested that MDM2 promotes cell motility and invasiveness by regulating E-cadherin ubiquitination and degradation, indicating that the E3 ligase activity of MDM2 might be involved in cancer metastasis. Nonetheless, there is no clear evidence of anti-metastasis activity due to the inhibition of MDM2 ubiquitin E3 ligase activity. In the contrast, the antagonists targeting MDM2-p53 binding exert excellent anti-metastasis effects. SP-141, a specific MDM2 inhibitor binding to the hydrophobic pocket occupied by $\mathrm{p} 53$, exerts potent therapeutic effects to decrease the tumour growth and inhibit metastasis in breast cancer models (Wang et al, 2014; Qin et al, 2016). 
A

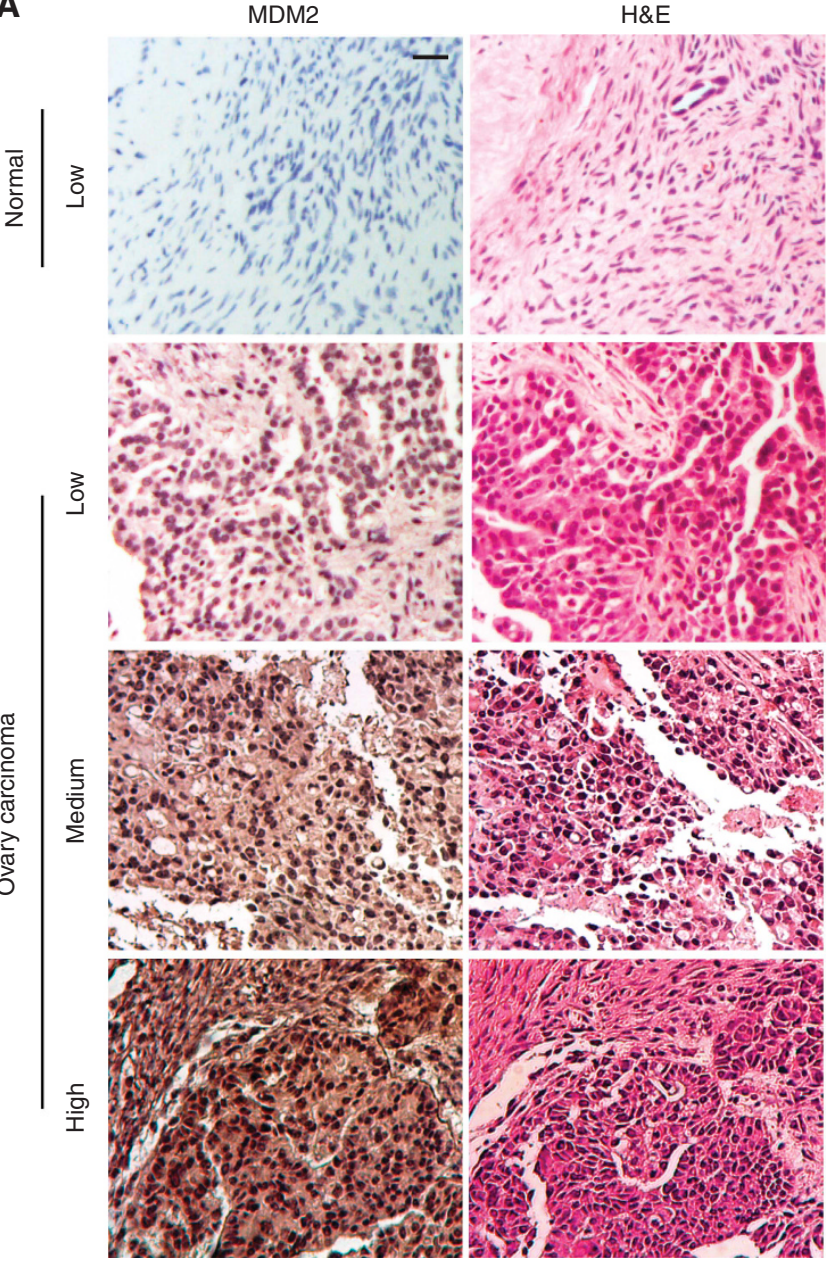

B

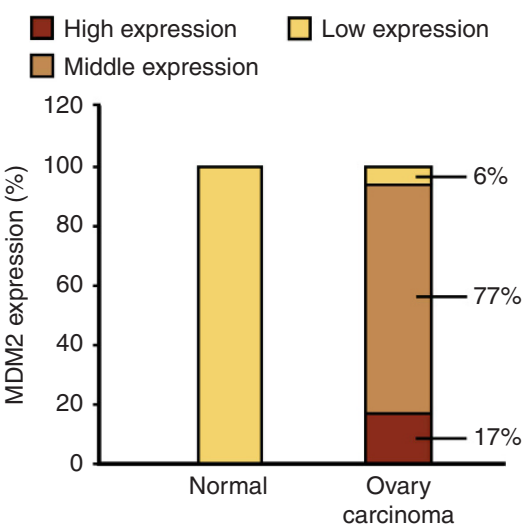

C

High expression

Low expression

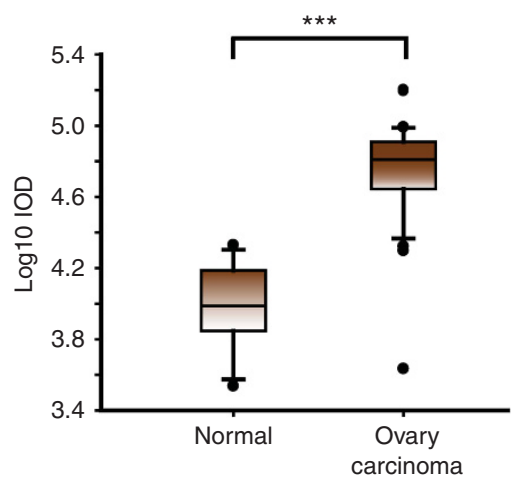

Figure 6. MDM2 expression is associated with the stage of ovary carcinoma. (A) Representative haematoxylin and eosin (H\&E) and immunohistochemical (IHC) staining images of MDM2 expression in human ovary carcinoma tissues compared with normal ovarian tissues, scale bar $=50 \mu \mathrm{m}$. (B) The IHC results of normal and ovary carcinoma tissues were divided into three groups and the percentages of different MDM2 expression levels were analysed by manual scoring method respectively: 1-4 (low expression); 5-8 (medium expression); and 9-12. (C) The expression scores of MDM2 in normal and ovary carcinoma tissues were measured and calculated by Image Pro Plus (IPP) image analysis software. Data were presented as box plot of the percent fourth-instar raw data. ${ }^{\star \star \star} P<0.001$, as evaluated using Student's $t$-test.

Table 1. The correlation of MDM2 expression and pathologic
feature
\begin{tabular}{|l|c|c|c|c|}
\hline & $\begin{array}{c}\text { Stage I-II } \\
\text { (no.) non- } \\
\text { metastasis }\end{array}$ & $\begin{array}{c}\text { Stage III-IV } \\
\text { (no.) } \\
\text { metastasis }\end{array}$ & $\chi^{2}$ & P-value \\
\hline Low expression & 5 & 1 & & \\
\hline Medium expression & 16 & 64 & 19.400 & $6.13 \mathrm{E}-05$ \\
\hline High expression & 0 & 18 & & \\
\hline
\end{tabular}

Furthermore, studies in renal cell carcinoma cells found that MDM2 promotes cell motility and invasiveness through a RING-finger independent mechanism (Polanski et al, 2010), implying that the pro-metastatic functions of MDM2 is not mediated by its E3 ligase activity.

Human MDM2 is a complicated and highly regulated protein comprising of 4 functional domains (Zhan et al, 2012; KarniSchmidt et al, 2016), among these regions, the N-terminal and the $\mathrm{C}$-terminal are most critical. The $\mathrm{N}$-terminal region of $\sim 100$ amino acid residues is essential for binding with high affinity to the other proteins like $\mathrm{p} 53$, for instance, thus regulating the functions of its client proteins. The C-terminus comprising a $\mathrm{Zn}^{2+}$-binding RING domain is essential for its E3 ubiquitin ligase activity. In our study, we found that the N-terminal depletion, but not the
C-terminal depletion of MDM2, lost the ability of inducing EMT and cell migration which was possessed by the full-length MDM2 (Figure 5F and G). Consist with this observation, we found Nutlin3, the MDM2 antagonist targeting N-terminus, repressed EMT by interfering with TGF- $\beta 1$-Smad Snail/Slug axis (Figure 5A and B); conversely, HLI-373, a MDM2 inhibitor interrupting its ubiquitin E3 ligase activity, failed to impose inhibitory effects on EMT and cell motility (Figure 5C-E). Thus, our findings reveal that the $\mathrm{N}$-terminus of MDM2 plays a critical role in inducing EMT and motility in ovarian cancer cells, serving Nutlin-3 as a potential therapeutic approach for ovarian cancer EMT and metastasis.

Mounting evidences have confirmed MDM2 is highly associated with cancer metastasis, yet limited studies have reported the correlation between MDM2 and cancer EMT. In recent studies, MDM2 has been shown to ubiquitinate E-cadherin, targeting it for degradation (Adhikary et al, 2014); or upregulate Snail expression subsequently to induce EMT both in breast cancer cells ( $\mathrm{Lu}$ et al, 2016). Nevertheless, whether MDM2 induces EMT progress in ovarian cancer remains unknown, since the role of MDM2 in EMT could be strictly cell type- and context-specific, owing to the negative regulation of MDM2 in lung cancer EMT, through MDM2-mediated degradation of Slug (Wang et al, 2009; Lin and Hsu, 2016). In this study, we provide for the first evidence indicating the involvement of MDM2 in ovarian cancer EMT. 
Because TGF- $\beta$ expresses aberrantly in ovarian cancer, leading to metastasis and malignancy, we introduced TGF- $\beta$ into ovarian cancer cells, to mimic pathological EMT progress. We found that TGF- $\beta$-induced EMT is reversible, impaired by the depletion of MDM2, while overexpression MDM2 enhanced EMT occurrence in ovarian cells, through TGF-Smads-Snail/Slug pathway (Figure 2). Moreover, we stated that the ability of MDM2 to induce EMT is its ubiquitin E3 ligase activity independent, different from the existing reports. Collectively, our findings demonstrated MDM2 could trigger EMT and may result in metastasis in ovarian cancer cells, providing a novel molecular target for developing ovarian cancer therapies.

Various studies uncovered that Smads played a crucial role in mediating the intracellular signalling of the TGF- $\beta$ (Macias et al, 2015 ) and the TGF- $\beta$-Smads pathway is a key growth regulatory signalling often aberrantly expressed in ovarian cancer and other malignancies (Chan et al, 2008). In the study of Yeung et al (2013), they showed TGF- $\beta$-Smads pathway modulates ovarian cancer invasion and EMT by upregulating target gene in tumour microenvironment. Thus, interfering the TGF- $\beta$-Smads pathway may be an effective strategy for metastasis ovarian cancer and lengthening survival durations in patients. It was reported that oncogene, MDM2, was induced by $\mathrm{Smad} 3 / \mathrm{Smad} 4$ complex in response to TGF- $\beta$, consequently leading to EMT and possible migration of breast cancer cells (Araki et al, 2010), indicating MDM2 is a downstream of TGF- $\beta$-Smads pathway. However, which whether could be mediated by MDM2 conversely remains unknown. Yam et al (1999) reported that MDM2 could suppress the transcriptional activity of ectopically expressed Smads, and they observed a colocalisation of MDM2 with ectopical Smads. In our present study, we focused on the function of endogenous Smads; and interestingly, MDM2 imposed positively regulatory effect on endogenous Smads, as indicated by enhanced phosphorylation of Smad2 or 3, induction of slug, snail, as well as suppression of E-cadherin (Figures 1-4). Conversely, the depletion of MDM2 resulted in downregulation of transcriptional activities of Smad complex, as well as the downstreaming targets including slug and snail (Figures 1-4). Together with the previous reports, a feed-back regulation of MDM 2 and TGF- $\beta$-Smads have been established, and may participate in the progression of EMT upon TGF- $\beta$ stimulation, further associated with decreased survival in ovarian cancer patients. This conclusion was in line with our analyses that the high expression of MDM2 and TGFB1 (the encoding gene of TGF- $\beta 1$ ) in ovarian cancer patients samples (obtained from the TGCA database) are correlated closely with the poor prognosis and shorter overall survival days (Supplementary Figure S6). Therefore, MDM2 may be a critical molecular participating in TGF- $\beta$-Smads pathway, regarded as a potent therapeutic target of metastasis ovarian cancer.

In summary, our present study suggested that through the activation of Smads pathway and the increased transcription of Snail/Slug, followed by the subsequent attenuation of E-cadherin, MDM2 enhanced the motility and EMT in ovarian cancer cells. Furthermore, the deletion of MDM2 N-terminus (1-109) and the antagonist targeting the N-terminus of MDM2, Nutlin-3, could impaired TGF- $\beta$ induced EMT and cell motility through TGF- $\beta$-Smads-Snail/Slug pathway (Supplementary Figure S7). Thus, the N-terminus of MDM2 is required for promoting cell motility and EMT occurrence and the MDM2 N-terminal selective antagonist may be regarded as potential anti-metastatic agents for ovarian cancer therapy.

\section{ACKNOWLEDGEMENTS}

This work was supported by the National Natural Science Foundation of China (81273536 and 81273534), National Natural
Science Foundation for Distinguished Young Scholar of China (81625024).

\section{CONFLICT OF INTEREST}

The authors declare no conflict of interest.

\section{REFERENCES}

Adhikary A, Chakraborty S, Mazumdar M, Ghosh S, Mukherjee S, Manna A, Mohanty S, Nakka KK, Joshi S, De A, Chattopadhyay S, Sa G, Das T (2014) Inhibition of epithelial to mesenchymal transition by E-cadherin up-regulation via repression of slug transcription and inhibition of E-cadherin degradation: dual role of scaffold/matrix attachment region-binding protein 1 (SMAR1) in breast cancer cells. J Biol Chem 289: 25431-25444.

Araki S, Eitel JA, Batuello CN, Bijangi-Vishehsaraei K, Xie XJ, Danielpour D, Pollok KE, Boothman DA, Mayo LD (2010) TGF-beta1-induced expression of human $\mathrm{Mdm} 2$ correlates with late-stage metastatic breast cancer. J Clin Invest 120: 290-302.

Bowtell DD, Bohm S, Ahmed AA, Aspuria PJ, Bast Jr RC, Beral V, Berek JS, Birrer MJ, Blagden S, Bookman MA, Brenton JD, Chiappinelli KB, Martins FC, Coukos G, Drapkin R, Edmondson R, Fotopoulou C, Gabra H, Galon J, Gourley C, Heong V, Huntsman DG, Iwanicki M, Karlan BY, Kaye A, Lengyel E, Levine DA, Lu KH, McNeish IA, Menon U, Narod SA, Nelson BH, Nephew KP, Pharoah P, Powell Jr DJ, Ramos P, Romero IL, Scott CL, Sood AK, Stronach EA, Balkwill FR (2015) Rethinking ovarian cancer II: reducing mortality from high-grade serous ovarian cancer. Nat Rev Cancer 15: 668-679.

Chan MW, Huang YW, Hartman-Frey C, Kuo CT, Deatherage D, Qin H, Cheng AS, Yan PS, Davuluri RV, Huang TH, Nephew KP, Lin HJ (2008) Aberrant transforming growth factor betal signaling and SMAD4 nuclear translocation confer epigenetic repression of ADAM19 in ovarian cancer. Neoplasia 10: 908-919.

Grochola LF, Taubert H, Greither T, Bhanot U, Udelnow A, Wurl P (2011) Elevated transcript levels from the MDM2 P1 promoter and low p53 transcript levels are associated with poor prognosis in human pancreatic ductal adenocarcinoma. Pancreas 40: 265-270.

Inoue K, Fry EA (2016) Aberrant splicing of the DMP1-ARF-MDM2-p53 pathway in cancer. Int J Cancer 139: 33-41.

Jayson GC, Kohn EC, Kitchener HC, Ledermann JA (2014) Ovarian cancer. Lancet 384: 1376-1388.

Karni-Schmidt O, Lokshin M, Prives C (2016) The roles of MDM2 and MDMX in cancer. Annu Rev Pathol 11: 617-644.

Lin TY, Hsu HY (2016) Ling Zhi-8 reduces lung cancer mobility and metastasis through disruption of focal adhesion and induction of MDM2-mediated Slug degradation. Cancer Lett 375: 340-348.

Lu X, Yan C, Huang Y, Shi D, Fu Z, Qiu J, Yin Y (2016) Mouse double minute 2 (MDM2) upregulates Snail expression and induces epithelial-tomesenchymal transition in breast cancer cells in vitro and in vivo. Oncotarget 7: 37177-37191.

Macias MJ, Martin-Malpartida P, Massague J (2015) Structural determinants of Smad function in TGF-beta signaling. Trends Biochem Sci 40: 296-308.

Matsumura N, Huang Z, Mori S, Baba T, Fujii S, Konishi I, Iversen ES, Berchuck A, Murphy SK (2011) Epigenetic suppression of the TGF-beta pathway revealed by transcriptome profiling in ovarian cancer. Genome Res 21: 74-82.

Narod S (2016) Can advanced-stage ovarian cancer be cured? Nat Rev Clin Oncol 13: 255-261.

Nieto MA, Huang RY, Jackson RA, Thiery JP (2016) Emt: 2016. Cell 166: 21-45.

Polanski R, Warburton HE, Ray-Sinha A, Devling T, Pakula H, Rubbi CP, Vlatkovic N, Boyd MT (2010) MDM2 promotes cell motility and invasiveness through a RING-finger independent mechanism. FEBS Lett 584: 4695-4702.

Qin JJ, Wang W, Sarkar S, Zhang R (2016) Oral delivery of anti-MDM2 inhibitor SP141-loaded FcRn-targeted nanoparticles to treat breast cancer and metastasis. J Control Release 237: 101-114.

Rosano L, Cianfrocca R, Tocci P, Spinella F, Di Castro V, Caprara V, Semprucci E, Ferrandina G, Natali PG, Bagnato A (2014) Endothelin A 
receptor/beta-arrestin signaling to the Wnt pathway renders ovarian cancer cells resistant to chemotherapy. Cancer Res 74: 7453-7464.

Shadfan M, Lopez-Pajares V, Yuan ZM (2012) MDM2 and MDMX: Alone and together in regulation of p53. Transl Cancer Res 1: 88-89.

Shi WD, Meng ZQ, Chen Z, Lin JH, Zhou ZH, Liu LM (2009) Identification of liver metastasis-related genes in a novel human pancreatic carcinoma cell model by microarray analysis. Cancer Lett 283: 84-91.

Siegel RL, Miller KD, Jemal A (2015) Cancer statistics, 2015. CA Cancer J Clin 65: 5-29.

Tsai JH, Yang J (2013) Epithelial-mesenchymal plasticity in carcinoma metastasis. Genes Dev 27: 2192-2206.

Wang SP, Wang WL, Chang YL, Wu CT, Chao YC, Kao SH, Yuan A, Lin CW, Yang SC, Chan WK, Li KC, Hong TM, Yang PC (2009) p53 controls cancer cell invasion by inducing the MDM2-mediated degradation of Slug. Nat Cell Biol 11: 694-704.

Wang W, Qin JJ, Voruganti S, Srivenugopal KS, Nag S, Patil S, Sharma H, Wang MH, Wang H, Buolamwini JK, Zhang R (2014) The pyrido[b]indole MDM2 inhibitor SP-141 exerts potent therapeutic effects in breast cancer models. Nat Commun 5: 5086.

Wu DW, Lee MC, Wang J, Chen CY, Cheng YW, Lee H (2014a) DDX3 loss by 553 inactivation promotes tumor malignancy via the MDM2/Slug/Ecadherin pathway and poor patient outcome in non-small-cell lung cancer. Oncogene 33: 1515-1526.

Wu Y, Fu Y, Zheng L, Lin G, Ma J, Lou J, Zhu H, He Q, Yang B (2014b) Nutlin-3 inhibits epithelial-mesenchymal transition by interfering with canonical transforming growth factor-beta1-Smad-Snail/Slug axis. Cancer Lett 342: 82-91.
Xu J, Lamouille S, Derynck R (2009) TGF-beta-induced epithelial to mesenchymal transition. Cell Res 19: 156-172.

Yam CH, Siu WY, Arooz T, Chiu CH, Lau A, Wang XQ, Poon RY (1999) MDM2 and MDMX inhibit the transcriptional activity of ectopically expressed SMAD proteins. Cancer Res 59: 5075-5078.

Yang JY, Zong CS, Xia W, Wei Y, Ali-Seyed M, Li Z, Broglio K, Berry DA, Hung MC (2006) MDM2 promotes cell motility and invasiveness by regulating E-cadherin degradation. Mol Cell Biol 26: 7269-7282.

Yeung TL, Leung CS, Wong KK, Samimi G, Thompson MS, Liu J, Zaid TM, Ghosh S, Birrer MJ, Mok SC (2013) TGF-beta modulates ovarian cancer invasion by upregulating CAF-derived versican in the tumor microenvironment. Cancer Res 73: 5016-5028.

Zhan C, Varney K, Yuan W, Zhao L, Lu W (2012) Interrogation of MDM2 phosphorylation in $\mathrm{p} 53$ activation using native chemical ligation: the functional role of Ser17 phosphorylation in MDM2 reexamined. $J$ Am Chem Soc 134: 6855-6864.

Zheng T, Wang J, Zhao Y, Zhang C, Lin M, Wang X, Yu H, Liu L, Feng Z, $\mathrm{Hu}$ W (2013) Spliced MDM2 isoforms promote mutant p53 accumulation and gain-of-function in tumorigenesis. Nat Commun 4: 2996.

This work is published under the standard license to publish agreement. After 12 months the work will become freely available and the license terms will switch to a Creative Commons AttributionNonCommercial-Share Alike 4.0 Unported License.

Supplementary Information accompanies this paper on British Journal of Cancer website (http://www.nature.com/bjc) 